\title{
POTENTIAL INEQUALITY REVISITED II: EQUALITY CASE AND HARDY TYPE INEQUALITIES
}

\author{
Neven Elezović, Josip PeČARić And MarJan PRALJAK
}

Abstract. A detailed analysis of potential inequality from [6] and [1] is presented. Special attention is given to the equality case. This enables us to obtain some improvements and generalizations of classical Hardy's inequality.

Mathematics subject classification (2010): 26D15.

Keywords and phrases: Potential inequality, potential equality, Hardy's inequality.

\section{REFERENCES}

[1] N. Elezović, J. PeČArić, M. Praljak, Potential inequality revisited, I: General case, Math. Inequal. Appl. 15, 4 (2012), 787-810.

[2] G. H. Hardy, J. E. Littlewood and G. Pólya, Inequalities, Cambridge University Press, 1934.

[3] E. Hewitt, K. Stromberg, Real and Abstract Analysis, Springer-Verlag, Heidelberg, 1975.

[4] A. Kufner, H. Triebel, Generalization of Hardy's inequality, Confer. Sem. Mat. Univ. Bari 156 (1978), 1-21.

[5] M. RaO, Brownian Motion and Classical Potential Theory, Lecture Notes Series No. 47, Aarhus University, 1977.

[6] M. RAO, H. ŠIKIĆ, Potential Inequality, Israel J. Math. 83 (1993), 97-127. 